\title{
Chromosomal microarray vs. NIPS: analysis of 5541 low-risk pregnancies
}

\author{
Lena Sagi-Dain, MD ${ }^{1}$, Lital Cohen Vig, $\mathrm{MD}^{2}$, Sarit Kahana, $\mathrm{PhD}^{3}$, Shiri Yacobson, $\mathrm{MSc}^{3}$, \\ Tamar Tenne, $\mathrm{PhD}^{4}$, Ifat Agmon-Fishman, $\mathrm{MSc}^{3}$, Cochava Klein, $\mathrm{Bsc}^{3}$, Reut Matar, $\mathrm{PhD}^{3}$, \\ Lina Basel-Salmon, MD, $\mathrm{PhD}^{3,5,6,7}$ and Idit Maya, $\mathrm{MD}^{3}$
}

Purpose: To evaluate the diagnostic yield of chromosomal microarray (CMA) in pregnancies with normal ultrasound.

Methods: This retrospective cohort analysis included all pregnancies with normal ultrasound undergoing CMA testing between the years 2010 and 2016. We calculated the rate of detection of clinically significant CMA findings in the whole cohort and according to various indications.

Results: Of $5541 \mathrm{CMA}$ analyses, clinically significant findings were yielded in 78 cases $(1.4 \%)$. Of these, 31 (39.7\%) variants could have theoretically been detected by karyotyping (e.g., sized above $10 \mathrm{Mb}$ ), and $28(35.9 \%)$ by noninvasive prenatal screening aimed at five common aneuploidies. Of the 47 submicroscopic findings detectable by CMA only, the majority (37 cases, $78.7 \%$ ) represented known recurrent syndromes. Detection of clinically significant CMA findings in women with no indication for invasive testing was
$0.76 \%$ (21/2752), which was significantly lower compared with $1.8 \%$ in advanced maternal age group (41/2336), $2.8 \%$ in abnormal biochemical serum screening (6/211), and $4.1 \%(10 / 242)$ in fetuses with sonographic soft markers.

Conclusion: Clinically significant CMA aberrations are detected in 1 of 71 pregnancies with normal ultrasound, and in 1 of 131 women with no indication for invasive testing. Thus, CMA might be recommended a first-tier test in pregnancies with normal ultrasound.

Genetics in Medicine (2019) 21:2462-2467; https://doi.org/10.1038/s41436019-0550-x

Keywords: chromosomal microarray analysis; karyotype; lowrisk pregnancies; noninvasive prenatal screening; prenatal diagnosis

\section{INTRODUCTION}

Prenatal genetic testing is currently executed by various methods. Noninvasive prenatal testing (NIPT) or screening (NIPS) technology is based on either massively parallel sequencing or single-nucleotide polymorphism (SNP) analysis of cell-free placental DNA in maternal plasma. ${ }^{1}$ Its reported sensitivity and specificity for the detection of trisomy 21 is higher than $99 \%$ (refs. ${ }^{2,3}$ )

Invasive prenatal testing is performed by amniocentesis or chorionic villi sampling. Karyotyping is a conventional cytogenetic test that has been used since the 1960s. It is based on a microscopic visualization of chromosome banding with a resolution of $5-10 \mathrm{Mb}$. Because the test is performed on dividing cells, the turnaround time is increased and there is a risk of culture artifacts. Moreover, the need for dividing cells might prevent analysis in situations in which cell viability is compromised (e.g., intrauterine fetal death). ${ }^{4}$

Chromosomal microarray (CMA) analysis is used to detect copy-number variants (CNVs) throughout the genome with a resolution down to $10 \mathrm{~kb}$. The analysis may be based either on comparative genomic hybridization (CGH) or SNP array technologies. ${ }^{5-7}$ Similar to karyotyping, an invasive procedure is currently required to obtain fetal cells, but the assay can be performed on DNA purified directly from fetal cells, without the need for cell culturing; thus results are obtained faster. ${ }^{5}$ In the past decade, CMA has become the first-tier test in the evaluation of patients with intellectual disability, autism, and multiple congenital anomalies, as its estimated diagnostic yield in these cases is $15-20 \%\left(\right.$ ref. $^{8}$ ). In the prenatal context, the detection rate of potentially pathogenic CNVs in pregnancies with abnormal sonographic findings ranges between $5 \%$ and $8.5 \%$, depending on the type and severity of the congenital malformation. ${ }^{5}$ Thus, CMA has become the primary recommended test in pregnancies with sonographic fetal structural anomalies (replacing conventional karyotype). ${ }^{9}$ It must be noted that in exome sequencing fetuses with structural abnormalities, normal CMA and karyotype results might reveal the putative genetic diagnosis in up to $24 \%$ of cases. ${ }^{10}$

\footnotetext{
${ }^{1}$ Genetics Institute, Carmel Medical Center, affiliated to the Ruth and Bruce Rappaport Faculty of Medicine, Technion - Israel Institute of Technology, Haifa, Israel; ${ }^{2}$ Schneider Children's Medical Center of Israel, Petah Tikva, Israel; ${ }^{3}$ Recanati Genetics Institute, Beilinson Hospital, Rabin Medical Center, Petach Tikva, Israel; ${ }^{4}$ The Genetics Institute, Meir Medical Center, Kfar Saba, Israel; ${ }^{5}$ Sackler Faculty of Medicine, Tel Aviv University, Tel Aviv, Israel; ${ }^{6}$ Felsenstein Medical Research Center, Rabin Medical Center, Petah Tikva, Israel; ${ }^{7}$ Pediatric Genetics Unit, Schneider Children's Medical Center of Israel, Petah Tikva, Israel. Correspondence: Lena Sagi-Dain (lena2303@gmail.com)
} 
Nevertheless, in low-risk pregnancies with normal ultrasound, no consensus has been reached concerning the optimal method of prenatal genetic testing. ${ }^{1-13}$ The detection rate of clinically significant CMA findings in such pregnancies ranges between $0.5 \%$ and $1.7 \%$ in different studies. ${ }^{11,14-16}$

The aim of the present study was to determine the rate of detection of clinically significant CMA findings (including noninvasive-detectable, karyotype-detectable, and submicroscopic variants) in a large cohort of pregnancies with normal ultrasound, according to various indications (e.g., advanced maternal age, abnormal serum screening for Down syndrome, or parental request).

\section{MATERIALS AND METHODS}

All prenatal samples of amniotic fluid or chorionic villi obtained by multiple genetic institutes and transferred to our laboratory for CMA testing between 2010 and 2016 were included. Pregnancies undergoing CMA due to various sonographic anomalies (e.g., anatomic defects, polyhydramnios, or disorders of fetal growth) were excluded. This study was approved by the Institutional Review Board of Rabin Medical Center.

From 2010 to 2013 genome coverage CMA was performed using CGH-based microarrays, whereas from 2013 to 2016 SNP-based microarray was used. DNA extraction and CMA procedures are described in the Appendix. The data were analyzed by Nexus Copy Number software 7.5 (BioDiscovery, El Segundo, CA, USA).

Following the guidelines of American College of Medical Genetics and Genomics for interpretation and reporting of postnatal constitutional $\mathrm{CNVs},{ }^{17}$ the results reported to the physicians were divided into three categories:

1. Clinically significant, defined as gross chromosomal aberrations, submicroscopic pathogenic CNVs, and CNVs of unclear clinical significance, likely pathogenic.

2. Variant of unknown significance (VOUS), defined as CNVs of unclear clinical significance, not otherwise specified (NOS), and known CNVs with clinical penetrance lower than $10 \%$ based on our updated literature review (such as duplications at 15q13.3, 16p11.2, and 16 p11.13 loci). ${ }^{18,19}$

3. Normal (including no CNVs, benign CNVs, and CNVs of unclear clinical significance, likely benign). In addition, this category included VOUS findings below the report threshold of $1 \mathrm{Mb}$ for deletions and $2 \mathrm{Mb}$ for duplications, in accordance with guidelines of the Israeli Society of Medical Genetics.

All clinically significant results were further classified into three subgroups:

1. Karyotype-detectable: chromosomal aberrations equal to or larger than $10 \mathrm{Mb}$

2. NIPS-detectable: trisomies 13, 18, 21, XXX, XXY, XYY, and monosomy $\mathrm{X}$
3. Submicroscopic findings (detectable only by CMA): CNVs less than $10 \mathrm{Mb}$ in size

Detection rate was calculated for each subgroup. The predicted detection rate of karyotyping and NIPS was calculated for each clinically significant CMA finding.

Further analyses were performed by indication for testing as follows:

1. No medical indication for testing (women aged $<35$ years, with normal nuchal translucency (i.e., below $3 \mathrm{~mm}$ ) and normal sonographic survey (including absence of soft markers), as well as normal Down syndrome biochemical screening tests

2. Advanced maternal age (same as above except for maternal age 35 years and up)

3. Abnormal first- or second-trimester biochemical screening (at a cutoff of 1:380 for Down syndrome, with normal ultrasound)

4. Soft markers (see Supplementary Table 1)

In cases of several indications, we used the one most likely to predict an abnormal test result: "soft markers" category > abnormal serum screening $>$ maternal age $>$ no indication.

For each indication, we calculated the rate of detection of clinically significant CMA findings or VOUS. CMA detection rate was compared with the expected rate of detection of karyotyping and NIPS.

Chi-square test with Yates' correction was used to compare rates of detection between groups. A $p$ value of less than 0.05 was considered significant.

\section{RESULTS}

During the study period, our hospital-based clinical laboratory analyzed 8716 prenatal CMA samples, of which 5541 (63.57\%) were in pregnancies with normal ultrasound at time of amniocentesis.

Overall, clinically significant CMA findings were detected in 78 samples (1.41\% [95\% CI 1.1-1.76]) (Table 1). Of those, 28 findings (36.4\% of abnormal results, or $0.5 \%$ of the overall cohort) could have theoretically been detected by both traditional karyotyping and NIPS (Table 2). Those involved $10(0.18 \%)$ common autosomal trisomies and $18(0.3 \%)$ sex chromosome aneuploidies. In particular, the findings included six $(0.11 \%)$ cases of trisomy 21 , one complete and one partial trisomy 18 (18p mosaicism sized $15.3 \mathrm{Mb}$ ), two cases of trisomy 13 mosaicism, seven $(0.13 \%)$ fetuses with Klinefelter syndrome (one of these in a mosaic form), six (0.11\%) cases of $47, \mathrm{XXX}$, and five (0.09\%) cases of complete/mosaic X0.

Three additional karyotype-detectable findings that could have been missed by NIPS included a mosaic chromosomal 2q duplication sized about $240 \mathrm{Mb}$, a 15q11.1-q13.3 duplication sized $12.3 \mathrm{Mb}$, and Xq26.2-q28 deletion sized $20 \mathrm{Mb}$. Thus, karyotyping could have detected 31 aberrations (detection rate of $0.56 \%$, encompassing $40.3 \%$ of clinically significant findings detectable by CMA). 
Table 1 Clinically significant CMA results in 5541 pregnancies with normal ultrasound

$\begin{array}{ll}\text { Number } & \text { Clinically significant copy-number } \\ \text { of cases } & \text { variants }\end{array}$

\begin{tabular}{|c|c|c|}
\hline 28 & Karyotype and NIPS detectable & \\
\hline 6 & $47, X X Y$ & Complete \\
\hline 1 & 47, XXY mosaicism & Complete \\
\hline 6 & $47, X X X$ & Complete \\
\hline 4 & Mosaicism XY/X0 or XXXO & Complete \\
\hline 1 & Turner syndrome $\mathrm{XO}$ & Complete \\
\hline 6 & Trisomy 21 & Complete \\
\hline 1 & Trisomy 18 & Complete \\
\hline 1 & Gain: 18p mosaicism (15.3 Mb) & Complete \\
\hline 2 & Trisomy 13 mosaicism & Complete \\
\hline 3 & Karyotype but not NIPS detectable & \\
\hline 1 & Gain: 2q mosaicism & Complete \\
\hline 1 & Gain: 15q11.1-q13.3 (12.3 Mb) & Complete \\
\hline 1 & Deletion: Xq26.2-q28 (20 Mb) & Complete \\
\hline 37 & $\begin{array}{l}\text { CMA-detectable only: known } \\
\text { syndromes }\end{array}$ & \\
\hline 1 & Deletion: 1q21 (1.3 Mb) & High \\
\hline 2 & Gain: 1q21.1 (0.368 Mb) & Variable \\
\hline 2 & Deletion: 3q29 (1.33-1.6 Mb) & High \\
\hline 1 & Gain: 7q11.23 (4.8 Mb) & High \\
\hline 1 & $\begin{array}{l}\text { Gain: } 7 q 11.23 \text { (1.4 Mb), gain: 1q21 } \\
(0.3 \mathrm{Mb})\end{array}$ & Variable \\
\hline 1 & Gain: 15q11.2-q13.2 (8 Mb) & High \\
\hline 5 & Gain: 16p11.2 (0.5-0.8 Mb) & Variable \\
\hline 1 & Gain: 16p11.2 (3.65 Mb) & Variable \\
\hline 3 & Deletion: 16p11.2 (0.598 Mb) & High \\
\hline 1 & Deletion: 16p12.2 (0.632 Mb) & High \\
\hline 1 & Deletion: 16p13.11-p12.3 (2.8 Mb) & High \\
\hline 1 & Gain: 17p12 (1.49 Mb) & Variable \\
\hline 5 & Deletion: 17q12 (1.4 Mb) & High \\
\hline 2 & Deletion: 22q11.21 (2.2-2.7 Mb) & High \\
\hline 1 & Deletion: 22q11.21 (0.425 Mb) & Variable \\
\hline 4 & Gain: 22q11.21 (2.6-2.9 Mb) & Variable \\
\hline 1 & Gain: 22q11.21 (4.2 Mb) & Variable \\
\hline 3 & Gain: 22q11.21 (1.5 Mb) & Variable \\
\hline 1 & Gain: 22q11.23 (1.3 Mb) & Variable \\
\hline 10 & $\begin{array}{l}\text { CMA-detectable only: unique } \\
\text { copy-number syndromes }\end{array}$ & \\
\hline 1 & Deletion: 7q11.22 (0.3 Mb) & - \\
\hline 1 & Gain: $11 \mathrm{p} 15.1(1.83 \mathrm{Mb})$ & - \\
\hline 1 & Deletion: 11q22.3 (3.1 Mb) & - \\
\hline 1 & $\begin{array}{l}\text { Gain: 18p11.32 (7.4 Mb), gain: 8p11 } \\
\text { (3.1 Mb) }\end{array}$ & - \\
\hline 1 & Gain: 21q22.3 (1.3 Mb) & - \\
\hline 1 & Deletion: Xp21.2-21.1 (0.251 Mb) & - \\
\hline 1 & Gain: Xp21.2-p21.1 (0.4 Mb) & - \\
\hline 1 & Deletion: Xp21.2 (0.4 Mb) & - \\
\hline 1 & Deletion: Xp22.31 (1.64 Mb) & - \\
\hline 1 & Uniparental disomy 5 & - \\
\hline
\end{tabular}

CMA chromosomal microarray, NIPS noninvasive prenatal screening.
Forty-seven CNVs $(60.3 \%$ of clinically significant CMA results, or $0.85 \%$ of the overall cohort) were sized below $10 \mathrm{Mb}$ and thus were defined as non-karyotype detectable. Of the 37 known syndromes, 17 (45.9\%) were characterized by high penetrance (above $40 \%$ ), 15 of these comprising loss of CNVs. The remaining 20 (54.1\%) were of variable penetrance (10-40\%), 19 of these gains of CNVs. The most common pathogenic variants involved CNVs at 22q11.21 locus (12 cases, $15.4 \%$ of the abnormal CMA results, or $0.22 \%$ of the entire cohort), 16p11.2 locus (six duplications and three deletions, detected at a frequency of $0.16 \%$ ), five cases of $17 \mathrm{q} 12$ deletion and four CNVs at the 1q21.1 locus (three duplications and one deletion).

A subgroup analysis of CMA results was performed by reasons for invasive testing (Fig. 1, Table 2).

Detection of clinically significant findings in no indication cohort was $0.76 \%$, significantly lower compared with other cohorts. Similarly, the proportion of karyotype detectable findings, variants with complete penetrance, and overall aberrations with complete and high penetrance was significantly lower in no indication group. On the other hand, the rate of submicroscopic CNVs did not differ between the groups, except for a significantly higher rate in the soft markers cohort.

\section{DISCUSSION}

At present, consensus has not yet been reached regarding the implementation of prenatal CMA in pregnancies with normal ultrasound. ${ }^{9}$ Clinical data on the utility of CMA in such pregnancies are important for decision-making by both physicians and parents. A recent meta-analysis by Srebniak et al. has summarized papers examining the prevalence of pathogenic submicroscopic CNVs in fetuses tested due to advanced maternal age or parental anxiety. ${ }^{20}$ It must be noted that one of the criteria for study inclusion was a known normal karyotype (or a clear exclusion of large genomic aberrations of $>10 \mathrm{Mb}$ ). The search yielded 19 relevant studies, while the analysis itself was based on the 10 largest studies (more than 300 pregnancies each), encompassing a pooled cohort of 10,614 fetuses. Overall, submicroscopic aberrations were detected in $0.84 \%$ of pregnancies with known normal karyotype. Of 10,314 pregnancies with reported onset/penetrance of the CNVs, in $0.37 \%$ an earlyonset syndromic disorder was found, in $0.30 \%$ susceptibility $\mathrm{CNV}$, and in $0.11 \%$ late-onset diseases. As there is no consensus about reporting the two latter types of CNVs in prenatal settings, the authors focused mainly on early-onset syndromic disorders, and concluded that a calculated prevalence of such disorders due to a submicroscopic aberration is 1 in 270 low-risk pregnancies. Separate analysis of the advanced maternal age group showed a $0.38 \%$ rate of early-onset syndromic findings (29/7597) versus $0.43 \%$ (13/ 2970 ) in the no indication group, a nonsignificant difference. The authors noted that it implies that submicroscopic findings are not age related. 
Table 2 CMA detection rates according to indication for invasive testing

\begin{tabular}{|c|c|c|c|c|c|c|}
\hline \multirow[t]{2}{*}{ CMA results category } & \multirow[b]{2}{*}{ Total } & \multicolumn{5}{|c|}{ Indication, $n(\%)$} \\
\hline & & $\mathrm{NI}$ & Other & AMA & MSS & SM \\
\hline Total & 5541 & $2752(49.7)$ & $2789(50.3)$ & $2336(42.2)$ & $211(3.8)$ & $242(4.4)$ \\
\hline Clinically significant & $78(1.4)$ & $21(0.76)$ & $57(2.0)^{\mathrm{a}}$ & $41(1.8)^{b}$ & $6(2.8)^{c}$ & $10(4.1)^{\mathrm{a}}$ \\
\hline \multicolumn{7}{|l|}{ CNV types } \\
\hline Submicroscopic CNVs & $47(0.85)$ & $15(0.55)$ & $32(1.15)^{d}$ & $23(0.98)$ & $2(0.97)$ & $7(2.93)^{e}$ \\
\hline Karyotype-detectable & $31(0.56)$ & $6(0.22)$ & $25(0.90)^{f}$ & $18(0.77)^{\mathrm{g}}$ & $4(1.89)^{h}$ & $3(1.24)^{i}$ \\
\hline NIPS-detectable & $28(0.50)$ & $4(0.15)$ & $24(0.86)^{j}$ & $17(0.73)^{k}$ & $4(1.9)^{\prime}$ & $3(1.24)^{\mathrm{m}}$ \\
\hline Common trisomies & $10(0.18)$ & $2(0.07)$ & $8(0.29)$ & $4(0.17)$ & $2(0.95)^{n}$ & $2(0.83)^{\circ}$ \\
\hline \multicolumn{7}{|l|}{ Penetrance } \\
\hline Complete & $31(0.56)$ & $6(0.22)$ & $25(0.90)^{p}$ & $18(0.77)^{\mathrm{q}}$ & $4(1.89)^{r}$ & $3(1.24)^{5}$ \\
\hline
\end{tabular}

AMA advanced maternal age, CMA chromosomal microarray, CNV copy-number variant, MSS abnormal maternal first- or second-trimester serum screening, NI no medical indication for CMA, NIPS noninvasive prenatal screening, SM soft markers, VOUS variant of unknown significance.

$\mathrm{a}-p=0.0001, \mathrm{~b}-p=0.0020, \mathrm{c}-p=0.0072, \mathrm{~d}-p=0.0216, \mathrm{e}-p=0.0002, \mathrm{f}-p=0.0021, \mathrm{~g} p=0.0063, \mathrm{~h} p=0.0037, \mathrm{i} p=0.0304, \mathrm{j}-p=0.0005, \mathrm{k} p=$ $0.0015, \mathrm{I} p=0.0014, \mathrm{~m} p=0.0143, \mathrm{n} p=0.0275, \mathrm{o} p=0.0350, \mathrm{p}-p=0.0009, \mathrm{q} p=0.0063, \mathrm{r} p=0.0037, \mathrm{~s} p=0.0304, \mathrm{t} p=0.0215, \mathrm{u} p=0.0344, \mathrm{v} p=0.0059$, $w-p=0.0167, x p=0.0118$, y $p=0.0012, z p=0.0261$, aa $p=0.0002$

Statistically significant differences compared to non-indicated group are marked in bold.

In our study, the rate of submicroscopic CNVs (detectable only by CMA) in the no indication group was $0.55 \%$, roughly half compared with the advanced maternal age cohort (0.98\%). Taking into account all submicroscopic CNVs in the meta-analysis of Srebniak et al. (and not only early-onset syndromes), roughly similar numbers are calculated: $0.57 \%$ $(17 / 2970)$ in the no indication group versus $1.03 \%(78 / 7597)$ in the advanced maternal age cohort, a statistically significant difference $(p=0.0349)$.

The overall risk for clinically relevant CMA findings in the no indication cohort, including karyotype-detectable aberrations, was $0.76 \%$. As might be expected, this rate was found to be significantly lower compared with the advanced maternal age cohort $(1.8 \%)$, pregnancies with abnormal serum screening $(2.8 \%)$, and soft markers (4.1\%). Studies examining the rate of abnormal CMA results in various prenatal sonographic anomalies usually use reference data of pregnancies with normal ultrasound as a control cohort for effective estimate calculation. ${ }^{21,22}$ This latter group is commonly defined as "low-risk." ${ }^{14}$ However, the percentage of abnormal CMA in reference pregnancies differs among various low-risk subgroups. In our article we present one such cohort, encompassing 2752 women with a truly low risk of aneuploidy (aged below 35 years, with normal nuchal translucency and sonographic survey, as well as normal Down syndrome biochemical screening tests). Even these "lowest-risk" women have about 1 in 131 risk $(0.76 \%)$ for clinically significant CMA findings.

The overall detection rate of clinically significant CMA findings in 5541 pregnancies with normal fetal nuchal translucency and normal sonographic survey was $1.4 \%$, or 1 in every 71 low-risk pregnancies. Of these, findings with complete and high penetrance can be found in 1 of every 123 pregnancies with normal ultrasound. One of every 117 fetuses of these pregnancies (47/5509) had a clinically significant CNV that could have been detected only by CMA. Theoretically, karyotyping would have missed up to twothirds $(60.3 \%)$ of the abnormal findings, while NIPS aimed at five common chromosomal aneuploidies would not detect $64.1 \%$ (50/78), and NIPS aimed only at trisomy 13/18/21 would not uncover $87.2 \%(68 / 78)$ of clinically significant CNVs. These numbers are even more prominent in "no indication" pregnancies. In this cohort, $71.4 \%$ of the anomalies would have been missed by routine karyotyping, and $81.0 \%$ to $90.4 \%$ by NIPS aimed at five and three chromosomes, respectively. These findings correlate with previous literature; for instance, according to Shani et al., of 171 invasive prenatal tests performed due to "maternal concern," two (1.2\%) clinically significant abnormal results were noted, neither of these involving the five chromosomes tested by NIPS. ${ }^{23}$

Major pitfalls of prenatal microarray testing include the need for an invasive procedure to obtain fetal cells and the detection of CNVs of unclear significance and of variable penetrance. However, a recent meta-analysis calculated the procedure-related risks of miscarriage for amniocentesis and chorionic villi sampling as $0.11 \%(1: 833)$ and $0.22 \%$ (1:455), respectively. ${ }^{24}$ These rates are both considerably lower compared with the $1.4 \%$ risk for abnormal genetic findings, implying that the benefits of invasive prenatal testing by CMA 


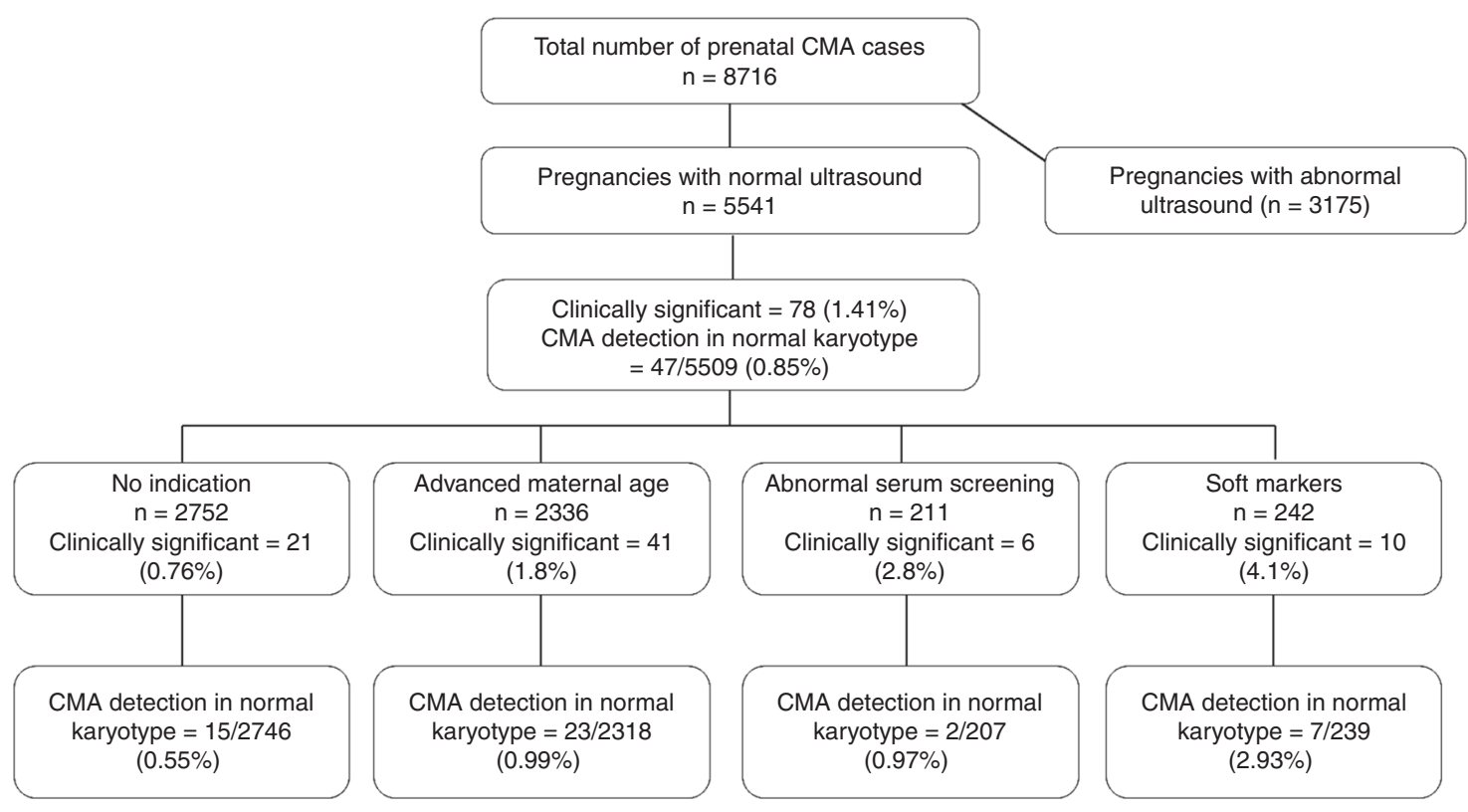

Fig. 1 Flow chart describing the distribution of the cohort and the CMA detection rate for each category. CMA chromosomal microarray.

seem to outweigh its limitations. In addition, invasive procedures allow performance of further genetic testing, such as exome sequencing.

As NIPS aimed only at the three common autosomal trisomies has replaced the traditional biochemical screening in several countries, ${ }^{25}$ it was interesting to show that such testing would detect only about $13 \%$ (10/78) of CMAdetectable variants in pregnancies with normal ultrasound, and only $9.5 \%(2 / 21)$ of these in women with no indication for prenatal testing. Expanded NIPS panels are now available for the detection of common microdeletion syndromes such as 1 p36 microdeletion, 4p deletion (Wolf-Hirschhorn syndrome), 5p deletion (cri du chat syndrome), 15q11.2-q13 (Angelman and Prader-Willi syndromes), and 22q11.21 microdeletion (DiGeorge syndrome). ${ }^{2}$ However, it must be noted that expanded NIPS aimed at these common loci (in conjunction with the five routine aneuploidies) would have missed almost half $(47.4 \%, 37 / 78)$ of the abnormal findings. Furthermore, about one-fifth (10/47) of the submicroscopic CNVs detected in our cohort were unique variants that would have been missed even by most expanded NIPS panels aimed at known pathogenic CNVs. Several publications have dealt with the utilization of genome-wide NIPS, and concluded that accuracy of such testing is influenced by sequencing depth, fetal DNA fraction, and CNV size. ${ }^{26,27}$ For instance, the sensitivity of a standard low-coverage genome sequencing ranged between $83 \%$ and $93.3 \%$ for CNVs larger than 5-6 Mb, dropping to $14.3-41.2 \%$ for variants sized between $1 \mathrm{Mb}$ and 5-6 Mb. ${ }^{26-28}$ Thus, several companies have set the detection cutoff of CNVs above $7 \mathrm{Mb}$. Furthermore, in low-risk pregnancies, the positive predictive value of NIPS for the detection of rare individual CNVs is much lower than in women at high risk for aneuploidy, estimated at about
$33-50 \%$ (refs. ${ }^{3,29}$ ), which could theoretically contribute to the rate of unnecessary invasive testing.

It could be argued that CMA testing may lead to a detection of VOUS findings, complicating genetic counseling and causing unnecessary stress for the parents. Hillman et al., in a systematic review and meta-analysis, reported a VOUS detection rate of about $1 \%$ (ref. $^{5}$ ), while in our study the rate of $0.4 \%$ was shown. This can be explained by the Israeli Society of Medical Genetics report threshold for VOUS: $1 \mathrm{Mb}$ for deletions and $2 \mathrm{Mb}$ for duplications. In addition, in Israel, couples are offered the option to not be informed about VOUS findings, which can reduce the burden of interpretation and management dilemmas of the pregnancy.

Limitations of our study include the retrospective nature of data acquisition, leading to lack of important parameters, such as later prenatal and postnatal follow-up. Nevertheless, this study presents a large cohort of truly low-risk women, for which CMA testing is informative and may have an additional diagnostic yield of $60-64 \%$ over NIPS and karyotyping. This study suggests that the risk for clinically significant CMA findings in low-risk pregnancies is greater than the current accepted risks of procedure-related miscarriage. Therefore, CMA could be considered a first-tier test in pregnancies with normal ultrasound, depending on the access to procedures, availability of appropriate pre- and post-test genetic counseling, as well as personal interpretation of risks and benefits of invasive procedures.

\section{SUPPLEMENTARY INFORMATION}

The online version of this article (https://doi.org/10.1038/s41436$019-0550-x)$ contains supplementary material, which is available to authorized users. 


\section{DISCLOSURE}

The authors declare no conflicts of interest.

Publisher's note: Springer Nature remains neutral with regard to jurisdictional claims in published maps and institutional affiliations.

\section{REFERENCES}

1. Neufeld-Kaiser WA, Cheng EY, Liu YJ. Positive predictive value of noninvasive prenatal screening for fetal chromosome disorders using cellfree DNA in maternal serum: independent clinical experience of a tertiary referral center. BMC Med. 2015;13:129.

2. Allyse $M$, Minear MA, Berson $E$, et al. Non-invasive prenatal testing: a review of international implementation and challenges. Int J Womens Health. 2015;7:113-126.

3. Committee opinion no. 640: cell-free DNA screening for fetal aneuploidy. Obstet Gynecol. 2015;126:e31-37.

4. Savage MS, Mourad MJ, Wapner RJ. Evolving applications of microarray analysis in prenatal diagnosis. Curr Opin Obstet Gynecol. 2011;23:103-108.

5. Hillman SC, McMullan DJ, Williams D, et al. Microarray comparative genomic hybridization in prenatal diagnosis: a review. Ultrasound Obstet Gynecol. 2012;40:385-391.

6. Committe opinion no. 581: the use of chromosomal microarray analysis in prenatal diagnosis. Obstet Gynecol. 2013;122:1374-1377.

7. Rajcan-Separovic E. Chromosome microarrays in human reproduction. Hum Reprod Update. 2012;18:555-567.

8. Miller DT, Adam MP, Aradhya S, et al. Consensus statement: chromosomal microarray is a first-tier clinical diagnostic test for individuals with developmental disabilities or congenital anomalies. Am J Hum Genet. 2010;86:749-764.

9. Practice bulletin no.162: prenatal diagnostic testing for genetic disorders. Obstet Gynecol. 2016;127:e108-122.

10. Fu F, Li R, Li Y, et al. Whole exome sequencing as a diagnostic adjunct to clinical testing in fetuses with structural abnormalities. Ultrasound Obstet Gynecol. 2018;51:493-502.

11. Lee CN, Lin SY, Lin CH, et al. Clinical utility of array comparative genomic hybridisation for prenatal diagnosis: a cohort study of 3171 pregnancies. BJOG. 2012;119:614-625.

12. ACOG committee opinion no. 446: array comparative genomic hybridization in prenatal diagnosis. Obstet Gynecol. 2009;114:1161-1163.

13. Novelli A, Grati FR, Ballarati L, et al. Microarray application in prenatal diagnosis: a position statement from the cytogenetics working group of the Italian Society of Human Genetics (SIGU), November 2011. Ultrasound Obstet Gynecol. 2012;39:384-388.

14. Fiorentino F, Napoletano S, Caiazzo F, et al. Chromosomal microarray analysis as a first-line test in pregnancies with a priori low risk for the detection of submicroscopic chromosomal abnormalities. Eur J Hum Genet. 2013;21:725-730.

15. Armengol L, Nevado J, Serra-Juhe C, et al. Clinical utility of chromosomal microarray analysis in invasive prenatal diagnosis. Hum Genet. 2012;131:513-523.

16. Wapner RJ, Martin CL, Levy B, et al. Chromosomal microarray versus karyotyping for prenatal diagnosis. N Engl J Med. 2012;367:2175-2184.

17. Kearney HM, Thorland EC, Brown KK, et al. American College of Medical Genetics standards and guidelines for interpretation and reporting of postnatal constitutional copy number variants. Genet Med. 2011;13:680-685.

18. Maya I, Sharony R, Yacobson S, et al. When genotype is not predictive of phenotype: implications for genetic counseling based on 21,594 chromosomal microarray analysis examinations. Genet Med. 2018; 20:128-131.

19. Rosenfeld JA, Coe BP, Eichler EE, et al. Estimates of penetrance for recurrent pathogenic copy-number variations. Genet Med. 2013;15:478-481.

20. Srebniak MI, Joosten M, Knapen M, et al. Frequency of submicroscopic chromosomal aberrations in pregnancies without increased risk for structural chromosomal aberrations: systematic review and meta-analysis. Ultrasound Obstet Gynecol. 2018;51:445-452.

21. Singer A, Maya I, Koifman A, et al. Microarray analysis in pregnancies with isolated echogenic bowel. Early Hum Dev. 2018;119:25-28.

22. Sagi-Dain L, Maya I, Falik-Zaccai T, et al. Isolated fetal horseshoe kidney does not seem to increase the risk for abnormal chromosomal microarray results. Eur J Obstet Gynecol Reprod Biol. 2018;222:80-83.

23. Shani H, Goldwaser T, Keating J, Klugman S. Chromosomal abnormalities not currently detected by cell-free fetal DNA: a retrospective analysis at a single center. Am J Obstet Gynecol. 2016;214:729 e721-729 e711.

24. Wulff CB, Gerds TA, Rode L, et al. Risk of fetal loss associated with invasive testing following combined first-trimester screening for Down syndrome: a national cohort of 147,987 singleton pregnancies. Ultrasound Obstet Gynecology. 2016;47:38-44.

25. van Schendel RV, van El CG, Pajkrt E, et al. Implementing noninvasive prenatal testing for aneuploidy in a national healthcare system: global challenges and national solutions. BMC Health Serv Res. 2017;17:670.

26. Yin $A H$, Peng $C F$, Zhao $X$, et al. Noninvasive detection of fetal subchromosomal abnormalities by semiconductor sequencing of maternal plasma DNA. Proc Natl Acad Sci USA. 2015;112:14670-14675.

27. Lo KK, Karampetsou E, Boustred C, et al. Limited clinical utility of noninvasive prenatal testing for subchromosomal abnormalities. Am J Hum Genet. 2016;98:34-44.

28. Li R, Wan J, Zhang $Y$, et al. Detection of fetal copy number variants by noninvasive prenatal testing for common aneuploidies. Ultrasound Obstet Gynecol. 2016;47:53-57.

29. Lutgendorf MA, Stoll KA. Why $99 \%$ may not be as good as you think it is: limitations of screening for rare diseases. J Matern Fetal Neonatal Med. 2016;29:1187-1189. 